University of Nebraska - Lincoln

DigitalCommons@University of Nebraska - Lincoln

February 1985

\title{
Piezomodulated Raman spectroscopy of molecular crystals: An experimental method for study of the anharmonic properties of solids
}

T. Luty

University of Nebraska - Lincoln

Craig J. Eckhardt

University of Nebraska - Lincoln, ceckhardt1@unl.edu

Follow this and additional works at: https://digitalcommons.unl.edu/chemistryeckhardt

Part of the Chemistry Commons

Luty, T. and Eckhardt, Craig J., "Piezomodulated Raman spectroscopy of molecular crystals: An experimental method for study of the anharmonic properties of solids" (1985). Craig J. Eckhardt Publications. 29.

https://digitalcommons.unl.edu/chemistryeckhardt/29

This Article is brought to you for free and open access by the Published Research - Department of Chemistry at DigitalCommons@University of Nebraska - Lincoln. It has been accepted for inclusion in Craig J. Eckhardt Publications by an authorized administrator of DigitalCommons@University of Nebraska - Lincoln. 


\title{
Piezomodulated Raman spectroscopy of molecular crystals: An experimental method for study of the anharmonic properties of solids ${ }^{a)}$
}

\author{
T. Luty and C. J. Eckhardt \\ Department of Chemistry, University of Nebraska, Lincoln, Nebraska 68588-0304
}

(Received 2 July 1984; accepted 29 August 1984)

\begin{abstract}
Consideration of phonon-phonon coupling induced by strain serves as impetus for developing a theoretical structure for piezomodulated Raman spectroscopy which can be used for experimental realization of the coupling. The direct relation of the coupling constant to generalized stress Gruneisen parameters is shown. These describe the crystal's anharmonic properties such as the thermal expansivity and the temperature dependence of the phonon energies. Numerical calculations are performed for anthracene crystal which exemplify the use of the stress Gruneisen parameters as sensitive measures of anisotropy of intermolecular interactions. Further development shows how the measurement can be used to determine these parameters and how piezomodulated Raman spectroscopy can be exploited to examine the anharmonicity of the lattice.
\end{abstract}

\section{INTRODUCTION}

Piezomodulation spectroscopy, where an oscillating stress is applied to a crystal and the optical response is synchronously detected, has proved to be a useful new technique for the study of molecular crystals. ${ }^{1}$ A theoretical study ${ }^{2}$ of spectroscopies based on dipole allowed transitions has shown that the piezomodulation spectroscopy locates both transverse and longitudinal frequencies of a band, provides information on internal strains, and gives direct measure of the partition of the mechanical energy among the various excitations of the crystal.

The efficacy of the technique in these spectroscopies suggests extension to other optical methods. Examination of inelastic light scattering techniques is of particular interest. Raman scattering is the archetype for such responses and, in conjunction with the piezomodulation, it may be expected to provide information on vibrational excitations in crystals.

Raman scattering is a powerful method for the study of lattice dynamics. For molecular crystals, the first-order scattering provides direct information about optical-phonon energies. Interpretation of such spectra usually deals with a symmetry assignment of observed phonon bands, only seldom with integrated intensities and very rarely with band shapes. For this reason, a common approximation is to treat optical, Raman-active phonons as noninteracting particles. However, since there are no truly harmonic solids, the phonons do interact among themselves and with other elementary excitations, and the effect is evident in the Raman spectra. Features such as temperature and pressure dependence of the Raman band shape and intensities, static stress-induced ${ }^{3}$ and orientational disorder-induced ${ }^{4,5}$ Raman scattering have already been investigated. The study of anharmonic processes in molecular crystals is a rapidly growing field and a substantial amount of work has been done recently. The phonon bandwidths and lifetimes can be directly mea-

\footnotetext{
a) Research supported by the Solid State Chemistry Program of the NSF through Grant DMR 79-08759.

b) Permanent address: Institute of Organic and Physical Chemistry, Technical University of Wroclaw, Poland.
}

sured $^{6,7}$ and, when compared with model calculations, provide very important and useful information about mechanisms of phonon-phonon interactions, thus making it also possible to refine the intermolecular potential at the anharmonic level ${ }^{8}$. In principle, all phonons can act as mediators for the phonon-phonon interactions, thus making the theoretical description ${ }^{9}$ and numerical calculations ${ }^{8}$ of the anharmonic processes very complicated. It has been shown that when interpreting phonon linewidths, ${ }^{6}$ the complexity can be avoided by assuming all cubic anharmonic terms (phonon-phonon coupling constants) to be equal. In this context, it would be useful to develop a selective spectroscopic method which will provide information about the phonon-phonon interactions mediated by particular kinds of phonons.

It is the aim of this paper to suggest that the piezomodulated Raman spectroscopy can serve as such a selective spectroscopy and measure coupling between Raman-active phonons due to acoustic phonons. Although the coupling does not fully account for the effect responsible for the phonon bandwidths, it is reasonable to expect that low-frequency acoustic phonons interacting with optical Ramanactive phonons will have an observable influence on band shapes and intensities. Thus the problem is to find out how to extract information about the interactions. In the case of statically stress-induced Raman experiments which have been done exclusively for inorganics, changes in phonon frequencies and their symmetries due to applied stress are of interest. For those materials, no change in intensities has been observed under nonresonant conditions, ${ }^{3}$ and there are no reports in the literature about band shape studies in those experiments. In order to extract information about phonon couplings due to applied stress, the relative changes in the Raman intensities have to be measured directly. Thus, in the method which is suggested here, the acoustic phonons are simulated as elastic waves produced by an applied modulated stress and the Raman intensities are measured synchronously with the applied perturbation. It should be stressed that there is growing interest in the use of acoustic phonons 
as a perturbation to probe couplings between elementary excitations in molecular crystals. In piezoreflection spectros$\operatorname{copy}^{1,2}$ and laser induced phonon spectroscopy, ${ }^{10}$ different aspects of coupling between electronic excitations and acoustic phonons are considered. Here, we shall consider interactions between optical phonons mediated by acoustic phonons which are generated by the applied modulated stress.

Two aspects must be considered: the dynamical problem and the magnitude of the response. The dynamical problem involves the lattice dynamics of a deformed crystal, while the problem of response will require the Raman scattering. Finally, a discussion will be given of how information about intermolecular interactions and some of the anharmonic properties of the lattice can be extracted from the experimental measurement.

\section{DYNAMICAL PROBLEM}

For the purpose of this paper, the acoustic phonons will be represented by strain components

$$
e_{\alpha \beta}=\lim _{q \beta \rightarrow 0} \bar{Q}_{\alpha}(q, j) q_{\beta},
$$

and optical phonons with frequencies $\omega_{0}(q, j)$ by normal coordinates $\bar{Q}(\mathbf{q}, j)$. The Hamiltonian for a strained lattice is

$$
\mathscr{H}=\mathscr{H}_{0}+\mathscr{H}^{\prime} \text {, }
$$

where $\mathscr{H}_{0}$ is the potential energy per cell of an undeformed lattice and

$$
\begin{aligned}
\mathscr{H}^{\prime}= & \frac{1}{2} v \sum_{\alpha \beta \gamma \delta} C_{\alpha \beta \gamma \delta}^{0} e_{\alpha \beta} e_{\gamma \delta}+\sum_{\alpha \beta} \sum_{j} \phi_{\alpha \beta j} e_{\alpha \beta} \bar{Q}(0, j) \\
& +\frac{1}{2 N} \sum_{\mathbf{q}} \sum_{j} \omega_{0}^{2}(\mathbf{q}, j) \bar{Q}(\mathbf{q}, j) \bar{Q}(-\mathbf{q}, j) \\
& +\frac{1}{2 N} \sum_{\alpha \beta} \sum_{\mathbf{q}} \sum_{\bar{j}} \phi_{\alpha \beta}\left(\mathbf{q}, j ;-\mathbf{q}, j^{\prime}\right) \\
& \times e_{\alpha \beta} \bar{Q}(\mathbf{q}, j) \bar{Q}\left(-\mathbf{q}, j^{\prime}\right)+\ldots
\end{aligned}
$$

Since the dynamical problem must be appropriate to the Raman experiment, simplification will be achieved by considering the zone-center optical modes only. The Hamiltonian $\mathscr{H}^{\prime}$ can then be renormalized by splitting $\bar{Q}(0, j)$ into two parts:

$$
\bar{Q}(0, j)=Q_{j}^{e l}+Q_{j}
$$

$Q_{j}^{e l}$ denotes the elastic displacement of molecules for a given strain $\{e\}$ and $Q_{j}$ is the vibrational part associated with this instantaneous equilibrium position of molecules such that $\left\langle Q_{j}\right\rangle=0$. Substitution into $\mathscr{H}^{\prime}$ and minimizing the Hamiltonian with respect to $Q_{j}^{e l}$ gives

$$
Q_{j}^{e l}=-\omega_{0 j}^{-2} \sum_{\alpha \beta} \phi_{\alpha \beta, j} e_{\alpha \beta}=\sum_{\alpha \beta} A_{\alpha \beta, j} e_{\alpha \beta},
$$

where only the linear term is retained. Substituting this expression into the Hamiltonian yields the effective Hamiltonian

$$
\begin{aligned}
\mathscr{H}^{\prime}= & \frac{1}{2} v \sum_{\alpha \beta \gamma \delta} C_{\alpha \beta \gamma \delta} e_{\alpha \beta} e_{\gamma \delta} \\
& +\frac{1}{2} \sum_{\not j}\left[\omega_{0 j}^{2} \delta_{j J}+\sum_{\alpha \beta} \phi_{\alpha \beta_{j j}} e_{\alpha \beta}\right] Q_{j} Q_{j} .
\end{aligned}
$$

The effective elastic constants are

$$
C_{\alpha \beta \gamma \delta}=C_{\alpha \beta \gamma \delta}^{0}-v \sum_{j} \omega_{0 j}^{-2} \phi_{\alpha \beta, j} \phi_{\gamma \delta, J}
$$

and they determine completely the elastic part of the potential energy. Equation (5) determines the internal strains due to an applied external one, and it is seen that the relation requires internal strains to be of the same symmetry as the $e_{\alpha \beta}$ component of the strain tensor.

At new equilibrium positions of the molecules, the dynamics are described by the second term in the Hamiltonian (6). The existence of the coupling between phonons $Q_{j}$ due to the applied strain $e_{\alpha \beta}$ and anharmonicity of the crystal implies that the solutions of the matrix

$$
\Omega_{i j^{\prime \prime}}=\omega_{0 j}^{2} \delta_{j j^{\prime}}+\sum_{\alpha \beta} \phi_{\alpha \beta_{i j} j^{\prime}} e_{\alpha \beta}
$$

are still harmonic phonons, but that the corresponding eigenvectors are no longer plane waves. The eigenfrequencies will depend upon the importance of this interaction and of its dependence on the strain. The symmetry of the coupling $\phi_{\alpha \beta, j}$. will determine which phonons $Q_{j}$ are coupled. For totally symmetric strains, phonons of the same symmetry are coupled while for nonsymmetric strains phonons of different symmetries are coupled. Denoting $\omega_{\gamma}^{2}$ as the eigenvalues of the matrix and $Q_{\gamma}$ as corresponding eigenvectors gives

$$
\mathscr{H}^{\prime}=\frac{1}{2} v \sum_{\alpha \beta \gamma \delta} C_{\alpha \beta \gamma \delta} e_{\alpha \beta} e_{\gamma \delta}+\frac{1}{2} \sum_{\lambda} \omega_{\lambda}^{2} Q_{\lambda}^{2} .
$$

The difference $\Sigma_{\lambda} \omega_{\lambda}-\Sigma_{j} \omega_{0 j}$ will be a measure of anharmonic interactions between optical phonons mediated by elastic waves, i.e., strain.

\section{OPTICAL RESPONSE}

The Raman-scattering intensity is governed by the tensor

$$
i_{\alpha \beta, \gamma \delta}(\omega)=\frac{1}{2 \pi} \int_{-\infty}^{\infty} d t \mathrm{e}^{\mathrm{i} \omega t}\left\langle\chi_{\gamma \delta}(\mathrm{t}) \chi_{\alpha \beta}(0)\right\rangle,
$$

where $\omega$ is the difference between scattered and incident light frequencies, $\chi_{\alpha \beta}$ are the tensor components of the electronic susceptibility of the crystal. To deal with Raman scattering from a strained crystal, the susceptibility is expanded in terms of strain components $e_{\gamma \delta}$ and the coordinates $Q(0, j)$,

$$
\begin{aligned}
\chi_{\alpha \beta}= & \chi_{\alpha \beta}^{0}+\sum_{j} \chi_{\alpha \beta, j} \bar{Q}(0, j)+\sum_{\gamma \delta} \chi_{\alpha \beta \gamma \delta} e_{\gamma \delta} \\
& +\sum_{j} \sum_{\gamma \delta} \chi_{\alpha \beta \delta \gamma, j} e_{\gamma \delta} \bar{Q}(0, j)+\cdots
\end{aligned}
$$

Using the substitution of Eq. (4) together with the equilibrium conditions (5), e.g.,

$$
\bar{Q}(0, j)=Q_{j}+\sum_{\gamma \delta} A_{\gamma \delta j} e_{\gamma \delta}
$$

and introducing it into Eq. (11) gives

$$
\begin{aligned}
\chi_{\alpha \beta}= & \chi_{\alpha \beta}^{0}+\sum_{j} \chi_{\alpha \beta, \rho_{j}} \\
& +\sum_{\gamma \delta}\left[\chi_{\alpha \beta \gamma \delta}+\sum_{j} \chi_{\alpha \beta, j} A_{\gamma \delta, j}\right] e_{\gamma \delta} \\
& +\sum_{\gamma \delta} \sum_{j} k_{\alpha \beta \gamma \delta j} Q_{j} e_{\gamma \delta}+\cdots
\end{aligned}
$$


The first coefficient of the expansion

$$
\chi_{\alpha \beta, j}=\frac{\partial \chi_{\alpha \beta}}{\partial Q_{j}}
$$

governs the first-order Raman scattering due to phonons with normal coordinates $Q_{j}$.

The photoelastic tensor component

$$
k_{\alpha \beta \gamma \delta}=\chi_{\alpha \beta \gamma \delta}+\sum_{j} \chi_{\alpha \beta, j} A_{\gamma \delta, j}
$$

has two contributions. $\chi_{\alpha \beta \gamma \delta}$ measures a change in the susceptibility component $\chi_{\gamma \beta}$ due to the external strain $e_{\gamma \delta}$, and is due to the change in the volume of the unit cell. The second corresponds to changes in the dielectric susceptibility component $\chi_{\alpha \beta}$ due to the internal strains. Finally, the last coefficient in Eq. (13) is defined as

$$
k_{\alpha \beta \gamma \delta, J}=\frac{\partial k_{\alpha \beta \gamma \delta}}{\partial Q_{j}} \text {. }
$$

An interesting result of Eq. (15) is that the contribution to the photoelastic tensor by the internal strains is determined by the same derivatives which govern the first-order Raman scattering. This result had first been noticed by Maradudin and Burstein ${ }^{11}$ for crystals of diamond structure and has been used recently ${ }^{12}$ for determination of the photoelastic constants of calcite. To our knowledge, no research in this area has been done for molecular solids. The reason may be attributed to the low symmetry of molecular crystals for which the theoretical approach of Maradudin and Burstein is not applicable. Here the problem is formulated in terms of normal coordinates of phonons and internal strains, thereby rendering the description more transparent for molecular solids. It is the internal strain which relates first-order Raman-scattering intensities to the photoelastic tensor. Due to recent theoretical studies of the internal strain in crystals $^{13,14}$, it is now possible to address this complicated problem of the photoelasticity of low-symmetry solids.

When the expansion of Eq. (13) is introduced into Eq. (10), the scattering intensity for a strained crystal is given by

$$
i(\omega)=i^{(1)}(\omega)+i^{(2)}(\omega)+i^{(3)}(\omega)
$$

where

$$
\begin{aligned}
& i_{\alpha \beta, \gamma \delta}^{(1)}(\omega)=\sum_{j j^{\prime}} \chi_{\alpha \beta, j} X_{\gamma \delta, j} X_{j j}^{\prime \prime}(\omega), \\
& i_{\alpha \beta, \gamma \delta}^{(2)}(\omega)=\sum_{j j^{\prime}} X_{\alpha \beta, j} \chi_{j j}^{\prime \prime},(\omega) \sum_{\eta \phi} k_{\gamma \delta \eta \phi J^{\prime}} e_{\eta \phi}, \\
& i_{\alpha \beta, \gamma \delta}^{(3)}(\omega)=\sum_{j j^{\prime}} \chi_{i j}^{\prime \prime}(\omega) \sum_{\eta \phi} k_{\alpha \beta \eta \phi, j} e_{\eta \phi} \sum_{\rho \tau} k_{\gamma \delta \rho \tau, j} e_{\rho \tau},
\end{aligned}
$$

and $\chi_{i j}^{\prime \prime}(\omega)$ stands for the imaginary part of the optical phonon-optical phonon correlation function

$$
\chi_{i j}^{\prime \prime}(\omega)=\frac{1}{2 \pi} \int_{-\infty}^{\infty} d t e^{\infty \omega t}\left\langle Q_{j}(t) Q_{f}(0)\right\rangle .
$$

When the phonons are treated as plane waves, the correlation function is nonzero only for $j=j^{\prime}$. However, due to the applied strain, the phonons do interact, and the correlation function for $j \neq j$ ' is not zero. In the derivation of Eqs. (17)(20), the slow time dependence of the external perturbative strain has been neglected. The other simplification is that the optical phonons have been treated as Einstein oscillators.

The susceptibility is now calculated for the coupled optical phonon-strain system. The Hamiltonian for the interacting system is

$$
\mathscr{H}=\frac{1}{2} \sum_{\not J j}\left[\omega_{0 j}^{2} \delta_{\nu l}+\sum_{\alpha \beta} \phi_{\alpha \beta, \not J} e_{\alpha \beta}\right] Q_{j} Q_{f^{\prime}}
$$

The single-particle susceptibility for noninteracting phonons systems can be defined as

$$
\chi_{j}^{0}(\omega)=\left\{\omega_{0 j}^{2}-\omega^{2}+i \omega \gamma_{j}\right\}^{-1},
$$

e.g., as for a damped harmonic oscillator with frequency $\omega_{0 j}$. Setting

$$
W_{j J^{\prime}}=\sum_{\alpha \beta} \phi_{\alpha \beta, j} e_{\alpha \beta},
$$

then from the definition of the generalized susceptibility of interacting systems ${ }^{15}$

$$
\chi_{i j^{\prime}}(\omega)=\left[1+W \cdot \chi^{0}(\omega)\right]_{j j}^{-1}, \chi_{j}^{0},(\omega),
$$

where $\chi^{0}(\omega)$ is a diagonal matrix with elements of noninteracting phonon susceptibilities $\chi_{j}^{\circ}(\omega)$. Equation (25) can be conveniently written as

$$
\chi_{i j^{\prime}}(\omega)=\left\{\left[\chi^{0}(\omega)\right]^{-1}+\mathbf{W}\right\}_{j j j^{-1}}^{-1} .
$$

The coupled system susceptibility will have poles at frequencies $\omega_{\lambda}$ [viz. Eq. (9)] appropriate to a deformed structure of the crystal, and the intensity in the Raman experiment will be given by $\mathrm{Eq}$. (17). In general, the intensity and maxima in the spectrum will depend on the applied strain. Experiments with inorganic materials under stress do not show changes in Raman intensities which would indicate that terms $i^{(2)}(\omega)$ and $i^{(3)}(\omega)$ are negligible in those solids. In order to obtain a useful result for the strain sensitivity of Raman scattering from molecular solids, a precise experimental technique will be required. This is possible with the use of differential measurements using piezomodulation. Here the stress is applied to a crystal and the response is measured synchronously with the modulation frequency. The first use was to study polymers, ${ }^{16}$ but without an underlying interpretation.

The piezomodulated Raman spectroscopy measures differences in scattering from a crystal in its compressed and extended deformation, or between a strained and unstrained crystal. In the first case, this corresponds to

$$
R^{\gamma \delta}(\omega)=\frac{\partial i(\omega)}{\partial \sigma_{\gamma \delta}} \cdot \sigma_{\gamma \delta}
$$

for a particular applied stress $\sigma_{\gamma \delta}$. Since the produced strain acts as a mediator for the phonon-phonon interactions, the equation has to be rewritten as

$$
R^{\gamma \delta}(\omega)=\sum_{\alpha \beta} \frac{\partial i(\omega)}{\partial e_{\alpha \beta}} \cdot\left(C^{-1}\right)_{\alpha \beta \gamma \delta} \cdot \sigma_{\gamma \delta},
$$

where $\mathrm{C}^{-1}$ is the inverse of the elastic constant tensor defined by Eq. (7). Apart from the term $\partial i(\omega) / \partial e_{\alpha \beta}$ in Eq. (28), it is seen that the piezomodulated Raman response will depend on the compressibility of a given crystal; the higher the compressibility, the larger the response will be. This is a promising result for molecular crystals are reasonably compressible and, in particular, it has interesting implications for ferroelastic materials where a softening of an elastic constant takes place close to a phase transition. 
The band shapes of the piezomodulated Raman spectra are described by the differential

$\frac{\partial \chi_{j j^{\prime}}^{\prime}(\omega)}{\partial e_{\alpha \beta}} \cdot e_{\alpha \beta} \sum_{j, j^{\prime \prime}} \chi_{j^{\prime \prime}}^{\prime \prime}(\omega) \phi_{\alpha \beta, j^{\prime \prime} j^{*}} \chi_{f^{\prime \prime \prime} j^{\prime \prime}}(\omega)$.

A simple example is a one-phonon oscillator with frequency $\omega_{0}$. The susceptibility of the strain-phonon system is

$$
\chi(\omega)=\left[1+\phi_{\gamma \beta} \cdot e_{\alpha \beta} \cdot \chi^{0}(\omega)\right]^{-1} \chi^{0}(\omega) .
$$

When the applied strain is very small, the susceptibility can be approximated as

$$
\chi(\omega)=\chi^{0}(\omega)-\chi^{0}(\omega) \phi_{\alpha \beta} e_{\alpha \beta} \chi^{0}(\omega),
$$

and the band shape of the piezomodulated Raman spectra will be determined by

$$
\frac{\partial \chi^{\prime \prime}(\omega)}{\partial e_{\alpha \beta}} \cdot e_{\alpha \beta}=\frac{2 \phi_{\alpha \beta} e_{\alpha \beta} \omega \gamma\left(\omega_{0}^{2}-\omega^{2}\right.}{\left[\left(\omega_{0}^{2}-\omega^{2}\right)^{2}+\omega^{2} \gamma^{2}\right]^{2}},
$$

e.g.,

$$
R^{\alpha \beta}(\omega)=\frac{\left(\omega_{+}^{2}-\omega_{-}^{2}\right) \omega \gamma\left(\omega_{0}^{2}\right)}{\left[\left(\omega_{0}^{2}\right)^{2}+\omega^{2} \gamma^{2}\right]^{2}}
$$

where from Eq. (8), $2 \phi_{\alpha \beta} e_{\alpha \beta}$ has been replaced by a difference in the phonon frequencies for the extended and compressed crystals. In the approximation, the zero-response is at $\omega=\omega_{0}$, e.g., at the frequency of the unstrained crystal, and the intensity depends on the coupling constant $\phi_{\alpha \beta}$. It is reasonable to assume that extrema in the derivative-like response correspond to $\omega_{+}$and $\omega_{-}$, but experimental verification must be obtained.

It is instructive to compare this simple result with the response for frequency modulated spectra. Here the band shape is determined by

$$
\frac{\partial \chi^{\prime \prime}(\omega)}{\partial \omega}=\frac{\left[\left(\omega_{0}^{2}-\omega^{2}\right)^{2}-\omega^{2} \gamma^{2}\right]-4 \omega^{2} \gamma\left(\omega_{0}^{2}-\omega_{2}\right)}{\left[\left(\omega_{0}^{2}-\omega^{2}\right)^{2}+\omega^{2} \gamma^{2}\right]^{2}} .
$$

When comparing Eq. (33) with Eq. (34), one sees that although the shapes of the modulated Raman spectra are predicted to be similar, the intensity will be different. Moreover, from the frequency modulated spectra, no physically interesting information will be extracted, while the strain modulated Raman spectra allows for extraction of the phononstrain coupling constant $\phi_{\alpha \beta}$. Since the coupling is due to the anharmonicity of the crystal, e.g., caused by higher terms in the energy expansion, more extensive investigation of the details of intermolecular interactions can be studied.

\section{RELATION OF THE COUPLING CONSTANT TO PHYSICAL PROPERTIES}

The Hamiltonian of Eq. (6) is the simplest anharmonic Hamiltonian from which an interpretation of the coupling constant can be gleaned. From the equation of motion of the Hamiltonian and upon taking an ensemble average, the following obtains:

$$
\left\langle e_{\alpha \beta}\right\rangle=\frac{1}{v} \sum_{\gamma \delta}\left(\mathrm{C}^{-1}\right)_{\alpha \beta \gamma \delta} \sum_{j,} \phi_{\gamma \delta, j,}\left\langle Q_{j} Q_{j}\right\rangle .
$$

To lowest order in the anharmonicity then,

$$
\left\langle Q_{j} Q_{j}\right\rangle=\left\langle Q_{j}^{2}\right\rangle \delta_{j j},=\frac{1}{\omega_{0 j}^{2}} \epsilon_{j}(T),
$$

where $\epsilon_{j}(T)$ is the energy of the phonon mode $j$.

The relation (35) now becomes

$$
\left\langle e_{\alpha \beta}\right\rangle=-\frac{1}{v} \sum_{\gamma \delta}\left(\mathrm{C}^{-1}\right)_{\alpha \beta \gamma \delta} \sum_{j} \frac{1}{\omega_{0 j}^{2}} \phi_{\gamma \delta, j} \cdot \epsilon_{j}(T),
$$

which can be interpreted as an averaged strain in the lattice due to the mean-square amplitudes of all thermally excited optical phonons $j$ that are coupled through the coupling constant $\phi_{\gamma \delta, j}$ to the strain $e_{\gamma \delta}$. The sum over $j$ in Eq. (37) can be also seen as a thermally activated stress

$$
\phi_{\gamma \delta}(T)=-\sum_{j} \phi_{\gamma \delta, j j}\left\langle Q_{j}^{2}\right\rangle,
$$

with $\phi_{\gamma \delta, i j}$ being a measure of how much a given thermally excited phonon mode $j$ contributes to the $\gamma \delta$-component of the stress. The stress results in an averaged strain which is in fact generated indirectly by internal strain $Q_{j}^{e l}$ [Eq. (5)]. This indirect coupling is already taken into account in the expression for the effective elastic constants [Eq. (7)] which are used in Eq. (37).

The thermally stimulated internal strain $\left\langle Q_{j}^{e l}\right\rangle$ can now be visualized in the following fashion. The thermally excited phonon mode $j$ with mean-square amplitude given by Eq. (35) drives the lattice strain [Eq. (37)]. The internal strains are then produced [Eq. (5)] to provide a necessary balance between homogeneous deformation and internal strains which follow from the condition for a minimum in the crystal energy.

The above interpretation is slightly different from that suggested in a recent paper ${ }^{17}$ where the main concern was focused on thermal expansion. This work provided an interesting relation between thermal expansion and the stresssensitivity of phonon modes. It serves as a straightforward application to information which may be obtained regarding the coupling constant.

Consistent with the approximation of Eq. (36) and from Eq. (8), it is assumed that

$$
\phi_{\gamma \delta, i j}=\frac{\partial \omega_{j}^{2}}{\partial e_{\gamma \delta}},
$$

and this is introduced into Eq. (37). From the definition of thermal expansion

$$
\alpha_{\alpha \beta}=\frac{d\left\langle e_{\alpha \beta}\right\rangle}{d T},
$$

the following is obtained:

$$
\alpha_{\alpha \beta}(T)=-\frac{1}{v} \sum_{j} \frac{\partial \ln \omega_{j}}{\partial \sigma_{\alpha \beta}} \cdot \mathrm{C}_{j}(T) .
$$

Here, $\mathrm{C}_{j}(T)$ is the contribution of the $j$ th phonon to the heat capacity, and quantity

$$
\gamma_{\alpha \beta}(j)=\frac{\partial \ln \omega_{j}}{\partial \sigma_{\alpha \beta}}
$$

is similar to the recently proposed ${ }^{17}$ generalized Gruneisen parameter. The importance of this is that it can be directly measured in the piezomodulated Raman experiment. Thus the experiment permits separate measurement of the anisotropic contributions of every phonon mode at the center of the Brillouin zone to the thermal expansion. Such informa- 
tion will be essential to understanding anomalous thermal expansion in many molecular crystals.

These contributions also show the anisotropy of the pressure influence on the phonon frequencies as

$$
\gamma(j)=\frac{\partial \ln \omega_{j}}{\partial p}=-\frac{1}{3} \sum_{\alpha \alpha} \gamma_{\alpha \alpha}(j) \text {. }
$$

Since the pressure dependence of phonon frequencies is thought to be an important measure of intermolecular interaction $^{18}$, there now is the potential for direct detection of anisotropic contributions $\gamma_{\alpha \beta}(j)$ which are essential to the understanding of molecular solids. Methods of analyzing piezomodulated Raman data to uncover information about the strength of the intermolecular interactions in different directions in the crystal are under development.

The Gruneisen parameter measured in piezomodulated Raman spectroscopy can be further used to analyze another purely anharmonic effect in crystals, namely, the temperature dependence of phonon frequencies. This contains, in general, two contributions ${ }^{19}$

$$
\left(\frac{\partial \omega}{\partial T}\right)_{p}=\left(\frac{\partial \omega}{\partial T}\right)_{V}+\left(\frac{\partial \omega}{\partial V}\right)_{T}\left(\frac{\partial V}{\partial T}\right)_{p} .
$$

The first term on the right-hand side is the "explicit" contribution; it reflects the effect of the change in the phonon occupation numbers for fixed equilibrium positions. The second term, the "implicit" contribution, reflects the effects of the change in equilibrium intermolecular spacings that accompany a change in temperature. The relative importance of the two terms is a matter of interest since it is required for an understanding of the effect of temperature on the phonon spectrum, as well as a measure of covalent contributions to crystal forces. In particular, the coefficient

$$
\eta=\left(\frac{\partial \omega}{\partial V}\right)_{T}\left(\frac{\partial V}{\partial T}\right)_{p}\left(\frac{\partial \omega}{\partial T}\right)_{p}^{-1}
$$

has a value close to one when the crystal forces are dominated by van der Waals or ionic forces, and tends toward zero when covalent forces dominate ${ }^{19}$. With the possibility of measurement of the stress dependence of the phonon frequencies, the coefficient can now be formulated anisotropically

$$
\eta_{\alpha \beta}(j)=-\frac{1}{v} \gamma_{\alpha \beta}(j) \bar{\gamma}_{\alpha \beta}(j) \cdot \mathrm{C}_{j}(T)\left(\frac{\partial \ln \omega_{j}}{\partial T}\right)_{p}^{-1},
$$

where $\left(\partial \ln \omega_{j} / \partial T\right)_{p}$ is found experimentally and

$$
\bar{\gamma}_{\alpha \beta}(j)=\frac{\partial \ln \omega_{j}}{\partial e_{\alpha \beta}}=\sum_{\gamma \delta} C_{\alpha \beta \gamma \delta} \gamma_{\gamma \delta}(j) .
$$

Equation (43) can also be written as

$$
\eta_{\alpha \beta}(j)=\alpha_{\alpha \beta}(j) \sum_{\gamma \delta} C_{\alpha \beta \gamma \delta}\left(\frac{d T}{d \sigma_{\gamma \delta}}\right)_{\omega},
$$

where $\alpha_{\alpha \beta}(j)$ is the $j$ th mode "contribution" to the thermal expansion component $\alpha_{\alpha \beta} . C_{\alpha \beta \gamma \delta}$ is the elastic constant component and

$$
\left(\frac{d T}{d \sigma_{\gamma \delta}}\right)_{\omega}=\left(\frac{\partial \omega}{\partial \sigma_{\gamma \delta}}\right)\left(\frac{\partial \omega}{\partial T}\right)^{-1}
$$

is interpreted as an increase in temperature which compensates a stress increase in the sense of canceling the effect on the phonon frequency and keeping $\omega$ constant. Equation (45) is an anisotropic generalization of the isotropic parameter introduced by Zallen. ${ }^{19}$

It has been pointed out by Munn ${ }^{20}$ that the strain Gruneisen parameters for molecular crystals contain information not only about the anharmonic part of the intermolecular potential but also about the harmonic part. The last contribution comes from the internal strain relaxation, the effect which is responsible for the second term in the expression (7) for the effective elastic constants. In this context, the possibility of extracting purely anharmonic contributions (coupling constants, $\phi_{\alpha \beta, j}$, from piezomodulated Raman experiments, as shown in the previous section, seems to be especially valuable.

\section{CALCULATIONS OF $\gamma_{\alpha \beta}(i)$}

Because there is no information available about the dependence of optical-phonon frequencies in molecular crystals on different components of the strain tensor, numerical calculations have been performed for the archetypical molecular crystal, anthracene. The "6-exp" atom-atom potential function with parameters from Williams ${ }^{21}$ has been employed. The reason for this choice of the potential function and parameters is that, as was concluded by Dorner et al., ${ }^{22}$ Chaplot et al., ${ }^{23}$ and Dumas et al., ${ }^{24}$ the lattice statics and dynamics calculations for anthracene crystal with Williams parameters give best agreement with the experimental phonon frequencies and the shape of the calculated phonon dispersion branches corresponds well with the measured curves. Chaplot et al ${ }^{23}$ concluded also that the agreement gives "...more confidence in the intermolecular potential function." Obviously, such a comparison between the calculated harmonic frequencies and the experimental data is based on the assumption that the experimental frequencies are harmonic in nature and the shifts due to crystal anharmonicities are negligible. Thus, it is advisable to refine the intermolecular potential at the anharmonic level. It has recently been done for naphthalene crystal ${ }^{8}$ based on calculations of the temperature dependence of the anharmonic frequency shifts and bandwidths of lattice phonons.

The important suggestion of this paper is that the stress Gruneisen parameters $\gamma_{\alpha \beta}(j)$ or the coupling constants $\phi_{\alpha \beta_{i j} j}$, which will be obtained from piezomodulated Raman experiments, can be used to refine the intermolecular potential. The calculations of the stress Gruneisen parameters for the anthracene crystal which we present below should then be considered as an illustration of the computational procedure and as a starting point for further improvement of the intermolecular potential when the experimental data from piezomodulated Raman-scattering measurements become available. It is important to mention that the Raman-active phonons in the anthracene crystal are especially suitable for the refinement of the potential as it has been shown ${ }^{23}$ that those modes are not influenced by low-energy intramolecular vibrations and they are therefore purely librational at the center of the Brillouin zone.

As the first step in the calculations of the stress Gruneisen parameters, the internal energy of the anthracene crystal has been minimized ${ }^{25}$ simultaneously with respect to seven parameters $(\mathrm{a}, \mathrm{b}, \mathrm{c}, \beta$ and Euler angles $\phi, \psi, \theta$ describing the orientation of the molecules). The parameters are listed in 
TABLE I. Crystal structure minimization of anthracene crystal calculated with Williams (Ref. 21) parameters and experimental values (Ref. 31).

\begin{tabular}{lcc}
\hline \multicolumn{1}{c}{ Parameter } & Calculation & Experiment \\
\hline$a(\AA)$ & 8.159 & 8.443 \\
$b(\AA)$ & 6.016 & 6.002 \\
$c(\AA)$ & 11.085 & 11.124 \\
$\beta$ (deg) & 123.97 & 125.60 \\
$\phi(\mathrm{deg})$ & 216.61 & 218.19 \\
$\psi(\mathrm{deg})$ & 60.01 & 59.24 \\
$\theta$ (deg) & 15.84 & 14.12 \\
\hline
\end{tabular}

Table I and compared with experimental values.

For the strain-free equilibrium structure, the lattice dynamics in the harmonic approximation is solved. The next step requires change of the unit-cell parameters according to a particular strain component and minimization of the lattice energy with respect to molecular orientations which are the only internal strains in the anthracene crystal. Only totally symmetric strains were considered to retain the monoclinic structure. Relations between unit-cell parameters and strain components as derived by Schlenker et $a{ }^{26}{ }^{26}$ have been used.

The calculations have been performed for every strain component equal to 0.02 , corresponding to a contraction of the lattice, and separately keeping other strains zero. After the minimization procedure, the equilibrium orientation of the molecules has been determined for the crystal experiencing a particular strain. The lattice-dynamical problem is thus solved for the new equilibrium structure. Calculated internal strains, expressed as changes in Euler angles, are listed in Table II.

The procedure of the calculations described above corresponds to that expressed by Eqs. (3)-(9). Phonon frequencies so calculated are the $\omega_{\lambda}$ in Eq. (9). Table III summarizes the results which indicate that the frequencies change a few wave numbers for $1 \%$ of applied strain. This indicates that a crystal such as anthracene is more sensitive to strain than inorganics. $^{3}$

The values of the strain-Gruneisen parameters $\bar{\gamma}_{\alpha \beta}(j)$ show a significant anisotropy which has a physical meaning
TABLE II. Internal strains in anthracene crystal calculated for totally symmetric external strains.

\begin{tabular}{lcccc}
\hline \hline & $e_{11}$ & $e_{22}$ & $e_{33}$ & $e_{13}$ \\
\hline$\frac{\partial \phi}{\partial e_{i j}}(\mathrm{deg})$ & 56.5 & -4.9 & -84.4 & -83.7 \\
$\frac{\partial \psi}{\partial e_{i j}}(\mathrm{deg})$ & 26.9 & -7.0 & 12.8 & -84.8 \\
$\frac{\partial \theta}{\partial e_{i j}}(\mathrm{deg})$ & 24.6 & 37.7 & -104.3 & -55.9 \\
\hline \hline
\end{tabular}

reflecting the anisotropy of intermolecular interactions. As discussed above, the calculation of $\bar{\gamma}_{\alpha \beta}(\eta)$ parameters is straightforward, although they are not directly measurable quantities.

In the experiment, a specific stress is applied to a crystal. In this manner, the stress-Gruneisen parameters are measured. Those parameters as defined by Eq. (4) can be calculated from

$$
\gamma_{\alpha \beta}(j)=\sum_{\gamma \delta} \mathrm{S}_{\alpha \beta \gamma \delta} \bar{\gamma}_{\gamma \delta}(j),
$$

where $S_{\alpha \beta \gamma \delta}$ are the elements of the elastic compliance tensor. To keep the calculations consistent within the same potential function model, the elastic constant tensor $C$ and its inverse $S$ have been calculated from dispersion of acoustic phonons in ten directions of the Brillouin zone. Results are shown in Table IV and they agree very well with experimental values at $100 \mathrm{~K} .{ }^{29}$ These calculations are for $0 \mathrm{~K}$. The stress Gruneisen parameters are found using Eq. (46) and are listed in Table V. It is evident that the $c^{*}$ direction in the anthracene crystal is, from the point of view of intermolecular interactions, different from directions in the $a b$ plane. It is the direction perpendicular to the cleavage plane and thus of weaker interactions that are known to lead to many physical properties which display anomalous behavior. The model calculations mirror this and it can be concluded that the stress sensitivity of phonon modes can provide help in understanding the anisotropy of molecular interactions in other, more complicated, molecular solids.

TABLE III. Optical-phonon frequencies $\left(=\mathrm{cm}^{-1}\right)$ of anthracene crystal calculated for the strain-free equilibrium structure compared with experimental values and calculated strain Gruneisen parameter.

\begin{tabular}{|c|c|c|c|c|c|c|}
\hline \multicolumn{3}{|c|}{ Mode frequencies } & \multicolumn{4}{|c|}{ Gruneisen parameters } \\
\hline & Expt. & Calc. & $\bar{\gamma}_{11}$ & $\bar{\gamma}_{22}$ & $\bar{\gamma}_{33}$ & $\bar{\gamma}_{13}$ \\
\hline$A_{g}$ & 49.0 & 43.4 & 2.42 & 1.50 & 10.71 & -2.65 \\
\hline$A_{g}$ & 82.1 & 90.0 & 4.50 & 2.56 & 4.22 & -5.56 \\
\hline$A_{g}$ & 132.1 & 146.9 & 4.22 & 5.10 & 0.00 & -2.72 \\
\hline$B_{g}$ & 56.0 & 52.0 & 2.88 & 1.06 & 9.23 & -5.58 \\
\hline$B_{g}$ & 70.0 & 77.0 & 4.54 & 3.05 & 2.06 & -3.44 \\
\hline$B_{g}$ & 140.0 & 148.9 & 4.06 & 3.79 & -0.91 & -4.40 \\
\hline$A_{u}$ & - & 39.0 & 4.23 & 3.59 & 6.92 & -6.41 \\
\hline$A_{u}$ & 107.1 & 119.8 & 5.38 & 3.46 & -0.25 & -4.88 \\
\hline $\boldsymbol{B}_{u}$ & 71.0 & 60.5 & 3.64 & 4.96 & 0.74 & -4.05 \\
\hline
\end{tabular}

2 Raman-active frequencies are taken from Ref. 27 and infrared active frequencies from Ref. 28 . All determined at $4 \mathrm{~K}$. 
TABLE IV. (A) Calculated elastic constant tensor elements for anthracene (kbar). (B) Calculated elastic compliance tensor elements for anthracene (in $10^{-2} \mathrm{kbar}^{-1}$ ).

\begin{tabular}{|c|c|c|c|c|c|c|}
\hline$\overline{(A)}$ & 135 & $\begin{array}{r}30 \\
145\end{array}$ & $\begin{array}{r}50 \\
71 \\
202\end{array}$ & $\begin{array}{r}0 \\
0 \\
0 \\
35\end{array}$ & $\begin{array}{r}52 \\
25 \\
-33 \\
0 \\
68\end{array}$ & $\begin{array}{r}0 \\
0 \\
0 \\
-9 \\
0 \\
32\end{array}$ \\
\hline (B) & 1.68 & $\begin{array}{l}0.39 \\
1.10\end{array}$ & $\begin{array}{r}-0.85 \\
-0.65 \\
1.17\end{array}$ & $\begin{array}{l}0 \\
0 \\
0 \\
3.08\end{array}$ & $\begin{array}{r}-1.84 \\
-1.02 \\
1.46 \\
0 \\
3.96\end{array}$ & $\begin{array}{l}0 \\
0 \\
0 \\
0.87 \\
0 \\
3.37\end{array}$ \\
\hline
\end{tabular}

\section{CONCLUSIONS}

This paper has demonstrated that piezomodulated Raman spectroscopy can be a new method for the study of intermolecular interactions in solids. The coupling constant $\phi_{\alpha \beta_{j} j}$ which can be measured by this experiment plays an important role in determining anharmonic properties of crystals such as the thermal expansion, temperature dependence of phonon energies, and anisotropy of intermolecular interactions.

Of perhaps greater import is that these coupling constants can play an essential role in the theory of phase transitions since they represent the anharmonic part of the freeenergy expansion which stabilizes the crystal after a structural phase transition. The coupling between totally symmetric strain and an order parameter always has to be present and in some cases will even be responsible for the transformation. Here, the so-called reentrant phase transition in malononitrile ${ }^{32}$ can serve as an example. According to the current theoretical model, ${ }^{30}$ the unusual reentrant nature of the phase transition is due to "anomalous thermal contraction of the cell dimension $a$, represented by strain component $e_{11}$." As it relates to the above discussion, this means that the coupling term $\phi_{11, j}, e_{11} Q_{j} Q_{j}$, is believed to be responsible for the phase transition since it is the coupling constant which determines the thermal expansivity of the crystal. More detailed analysis of the phase transition in terms of the coupling constant is underway.

The approach to the problem of piezomodulated Raman spectroscopy has employed the following simplifications: (i) optical phonons are approximated by Einstein oscillators and (ii) applied stress is considered as homogeneous. When these approximations are lifted, the description will be more complicated, but can bring new insight. It can be expected that when the dispersion of the optical phonons is taken into account, the coupling constant will depend on an integration of the phonon states within the Brillouin zone and consequently the coupled system susceptibility will convey information about the phonon densities of states.

A qualitatively new problem will appear when the second approximation is lifted. Allowing the strain to propagate through the crystal with dissipation will force changes in the phonon densities of states. Measurement of the optical-phonon energies as a function of distance from the sur-
TABLE V. Stress Gruneisen parameters $\gamma_{\alpha \beta}(\eta)$ for optical-phonon modes in anthracene crystal $\left(\mathrm{kbar}^{-1}\right)$.

\begin{tabular}{lrcrrr}
\hline \hline Mode & \multicolumn{1}{c}{$\omega_{j}$} & $\gamma_{11}$ & \multicolumn{1}{c}{$\gamma_{22}$} & \multicolumn{1}{c}{$\gamma_{33}$} & \multicolumn{1}{c}{$\gamma_{13}$} \\
\hline $\boldsymbol{A}_{\mathrm{g}}$ & 43.4 & 0.004 & -0.017 & 0.057 & -0.008 \\
$\boldsymbol{A}_{\mathrm{g}}$ & 90.0 & 0.152 & 0.075 & -0.067 & -0.267 \\
$\boldsymbol{A}_{\mathrm{g}}$ & 146.9 & 0.141 & 0.100 & -0.151 & -0.237 \\
$\boldsymbol{B}_{\mathrm{g}}$ & 52.0 & 0.075 & 0.020 & -0.005 & -0.150 \\
$\boldsymbol{B}_{\mathrm{g}}$ & $\mathbf{7 7 . 0}$ & $\mathbf{0 . 1 2 9}$ & 0.069 & -0.078 & -0.213 \\
$\boldsymbol{B}_{\mathrm{g}}$ & 148.9 & 0.171 & 0.108 & -0.134 & -0.301 \\
$\boldsymbol{A}_{\mathrm{u}}$ & 39.0 & 0.144 & 0.082 & -0.072 & -0.267 \\
$\boldsymbol{A}_{\mathrm{u}}$ & 119.8 & 0.196 & 0.109 & -0.142 & -0.331 \\
$\boldsymbol{B}_{\mathrm{u}}$ & $\mathbf{6 0 . 5}$ & 0.148 & 0.105 & -0.114 & -0.267 \\
\hline \hline
\end{tabular}

face where the stress has been applied will provide a measure of optical-phonon velocities. Furthermore, the phonons will condense in a region of the crystal where the applied stress allows them to have lower energy. This can provide an excellent opportunity to study the details of structural phase transition mechanisms which are known to be based on phonon condensation.

'J. Merski and C. J. Eckhardt, J. Chem. Phys. 75, 3691, 3705, 3719, 3731 (1981).

${ }^{2}$ T. Luty and C. J. Eckhardt, J. Chem. Phys. 81, 520 (1984).

${ }^{3}$ E. M. Anastassakis, in Morphic Effects in Lattice Dynamics in Dynamical Properties of Solids, edited by G. K. Horton and A. A. Maradudin (NorthHolland, Amsterdam, 1980), Vol. 4.

${ }^{4}$ T. Geisel and J. Keller, J. Chem. Phys. 62, 3777 (1975).

${ }^{5}$ R. M. Pick, in Vibrational Spectroscopy of Molecular Liguids and Solids, edited by S. Bratos and R. M. Pick (Plenum, New York, 1980).

${ }^{6}$ C. L. Schosser and D. D. Dlott, J. Chem. Phys. 80, 1369 (1984)

${ }^{7}$ C. L. Schosser and D. D. Dlott, J. Chem. Phys. 80, 1394 (1984).

${ }^{8}$ R. G. Della Valle, P. F. Fracassi, R. Righini, and S. Califano, Chem. Phys. 74, 179 (1983).

${ }^{9}$ S. Califano, V. Schettino, and N. Neto, Lattice Dynamics of Molecular Crystals, Springer Series on Lecture Notes in Chemistry (Springer, Berlin, 1981), Vol. 26.

${ }^{10}$ M. D. Fayer, Annu. Rev. Phys. Chem. 33, 63 (1982).

${ }^{11}$ A. A. Maradudin and E. Burstein, Phys. Rev., 164, 1081 (1967).

${ }^{12}$ G. S. Kumari and N. R. Rao, Phys. Rev. B 28, 2172 (1983).

${ }^{13}$ E. M. Anastassakis and M. Cardona, Phys. Status Solidi B 104, 589 (1981).

${ }^{14}$ T. Luty and R. W. Munn, J. Phys. C 15, 4459 (1982).

15J. C. Raich, H. Yasuda, and E. R. Bernstein, J. Chem. Phys. 78, 6209 (1983).

${ }^{16}$ C. Tzimis, S. Bahi, P. Davidson, W. Risen, Jr., and R. Baughman, Rev. Sci. Instrum. 49, 1725 (1978).

${ }^{17}$ C. L. Choy, S. P. Wong, and K. Young, Phys. Rev. B 29, 1741 (1984).

${ }^{18}$ R. Zallen, Phys. Rev. B 9, 4485 (1974).

${ }^{19} R$. Zallen and M. L. Slade, Phys. Rev. B 18, 5775 (1978).

${ }^{20}$ R. W. Munn, Phys. Rev. B 12, 3491 (1975).

${ }^{21}$ D. E. Williams, J. Chem. Phys. 47, 4680 (1967).

${ }^{22}$ B. Dorner, E. L. Bokhenkov, S. L. Chaplot, J. Kalus, I. Natkamiec, G. S. Pawley, U. Schmeltzer, and E. F. Sheka, J. Phys. C 15, 2353 (1982).

${ }^{23}$ S. L. Chaplot, G. S. Pawley, B. Dorner, V. K. Jindal, J. Kalus, and I. Natkaniec, Phys. Status Solidi B 110, 445 (1982).

${ }^{24}$ G. Dumas, M.-P. Chedin, and F. Vovelle, J. Phys. (Paris) 41, 905 (1980).

${ }^{25}$ F. James and A. Ross, Program MINUIT, Cern Computer Center Program Library, D506, D516.

26. L. Schlenker, G. V. Gibbs, and M. B. Boiseu, Jr., Acta Crystallogr. Sect. A 34, 52 (1978).

${ }^{27}$ M. Suzuki, T. Yokoyama, and M. Ito, Spectrochim. Acta Sect. A 24, 1091 (1968).

${ }^{28} \mathrm{M}$. Hineo and H. Yoshinaga, Spectrochim. Acta Sect. A 31, 617 (1975).

${ }^{29}$ G. K. Afanas'eva and R. M. Myasnikova, Sov. Phys.-Crystallogr. 13, 921 (1969).

${ }^{30}$ A. I. M. Rae and M. T. Dove, J. Phys. C 16, 3233 (1983).

${ }^{31}$ R. Mason, Acta Crystallogr. 17, 547 (1964).

${ }^{32}$ N. LeCalvé, B. Pasquier, and A. Novak, J. Chem. Phys. 72, 6409 (1980). 